\title{
Miscellaneous observations of active galactic nuclei. II. ${ }^{\star}$
}

\author{
A.C. Gonçalves, P. Véron, and M.-P. Véron-Cetty \\ Observatoire de Haute Provence (CNRS), F-04870 Saint Michel l'Observatoire, France
}

Received March 20; accepted April 21, 1997

\begin{abstract}
We observed 37 AGN candidates and classified them on the basis of their spectroscopic properties; three are confirmed QSOs, one is a BL Lac object, nine are Seyfert 1 galaxies, four Seyfert 2s, while twenty are HII regions.
\end{abstract}

Key words: galaxies: active — galaxies: Seyfert galaxies: BL Lacertae objects: general - galaxies: quasars: general — galaxies: starburst

\section{Introduction}

In the course of several observing runs, we obtained optical spectra of 37 AGN candidates with uncertain classification. Twenty of them turned out to be extragalactic HII regions ionized by hot stars, while seventeen were confirmed to be QSOs, BL Lac or Seyfert galaxies. In a previous paper (Véron-Cetty \& Véron 1986), the classification of 61 AGN candidates was given.

\section{Observations}

Most of the observations were carried out in 1995, 1996 and 1997 with the spectrograph CARELEC (Lemaitre et al. 1989) attached to the Cassegrain focus of the Observatoire de Haute Provence (OHP) $1.93 \mathrm{~m}$ telescope. The spectrograph settings used during these runs are given in Table 1 . The detector was a $512 \times 512$ pixels, $27 \times 27 \mu \mathrm{m}$ Tektronic CCD. The slit width was 2.1 arcsec, corresponding to a projected slit width on the detector of $52 \mu \mathrm{m}$, or 1.9 pixel. The resolution, as measured on the night sky emission lines, was 13.5 and $3.4 \AA F W H M$ at low and high resolution, respectively. The spectra were flux calibrated using the standard stars given in Table 1, taken from Oke (1974), Stone (1977), Oke \& Gunn (1983) or Massey et al. (1988).

Send offprint requests to: P. Véron

* Based on observations collected at the Observatoire de Haute-Provence (CNRS), France, and at the European Southern Observatory, La Silla, Chili.
Additional observations were made with EFOSC (Dekker et al. 1988) at the $3.6 \mathrm{~m}$ ESO telescope in La Silla during two runs in July 1995 and August 1996. The detector was the CCD ESO\#26, similar to the one used at OHP. The dispersion was $230 \AA \mathrm{mm}^{-1}$; the slit width was 1.5 arcsec, corresponding to 2.2 pixels and resulting in a resolution of $15 \AA$. The wavelength range was $\lambda \lambda 3500-7600 \AA$. The spectra were flux calibrated using the standard stars W 485A and GD 190 (Oke 1974).

Table 1. Spectrograph settings and standard stars during OHP observations

\begin{tabular}{|c|c|c|c|}
\hline$\overline{\text { Date }}$ & $\begin{array}{c}\text { Dispersion } \\
\left(\AA \mathrm{mm}^{-1}\right)\end{array}$ & $\begin{array}{c}\lambda \text { Range } \\
(\AA)\end{array}$ & $\begin{array}{l}\text { Standard } \\
\text { stars }\end{array}$ \\
\hline $21-23.03 .95$ & 66 & $6500-7400$ & $\mathrm{BD} 26^{\circ} 2606$ \\
\hline $24-28.08 .95$ & 260 & $4500-8000$ & $\begin{array}{ll}\mathrm{BD} & 25^{\circ} 3941 \\
\mathrm{BD} & 28^{\circ} 4211\end{array}$ \\
\hline $28-31.08 .95$ & 66 & $6700-7600$ & $\begin{array}{l}\text { BD } 25^{\circ} 3941 \\
\text { BD } 28^{\circ} 4211\end{array}$ \\
\hline $31.08-04.09 .95$ & 66 & $4860-5760$ & $\begin{array}{l}\text { Feige } 15 \\
\text { BD } 28^{\circ} 4211\end{array}$ \\
\hline 10.05 .96 & 66 & $6700-7600$ & $\begin{array}{l}\text { GD } 140 \\
\text { BD } 26^{\circ} 2606\end{array}$ \\
\hline 13.05 .96 & 66 & $4860-5760$ & $\begin{array}{l}\text { Feige } 98 \\
\text { Kopff } 27\end{array}$ \\
\hline 08.06 .96 & 66 & $4860-5760$ & $\begin{array}{l}\text { Feige } 66 \\
\text { Kopff } 27\end{array}$ \\
\hline 09.06 .96 & 66 & $6700-7600$ & $\begin{array}{l}\text { Feige } 66 \\
\text { BD } 28^{\circ} 4211\end{array}$ \\
\hline $06-08.01 .97$ & 66 & $4720-5620$ & EG 247 \\
\hline $08-11.01 .97$ & 66 & $6175-7075$ & EG 247 \\
\hline
\end{tabular}

The journal of observations is given in Table 2 and the list of the observed objects with relevant data, in Table 3. The spectra were analysed in terms of Gaussian components as described in Véron et al. (1997). Table 4 gives for each object the velocity, width and relative strength of each line, together with the adopted classification. Objects with broad Balmer lines were classified as Seyfert 1 galaxies, or QSOs whenever their absolute magnitude was brighter than $M_{B}=-23.0$ (assuming $H_{0}=$ $50 \mathrm{~km} \mathrm{~s}^{-1} \mathrm{Mpc}^{-1}$ ); Seyfert 2s and HII regions were distinguished on the basis of the value of the $[\mathrm{OIII}] \lambda 5007 / \mathrm{H} \beta$ and $[\mathrm{NII}] \lambda 6584 / \mathrm{H} \alpha$ line ratios (Veilleux \& Osterbrock 
Table 2. Journal of observations. A: OHP, $260 \AA \mathrm{mm}^{-1}$; B: OHP, $66 \AA \mathrm{mm}^{-1}$ blue; C: OHP, $66 \AA \mathrm{mm} \mathrm{m}^{-1} \mathrm{red}$; D: ESO, $230 \AA \mathrm{mm}^{-1}$. An "** " after the exposure time indicates the presence of clouds during the exposure

\begin{tabular}{|c|c|c|c|c|c|c|c|}
\hline$\overline{\text { Name }}$ & Disp. & Date & $\begin{array}{l}\text { Exp. time } \\
\text { (min) }\end{array}$ & Name & Disp. & Date & $\begin{array}{l}\text { Exp. time } \\
\text { (min) }\end{array}$ \\
\hline$\overline{4 C ~ 12.05}$ & $\overline{\mathrm{D}}$ & 10.08 .96 & 10 & PKS $1420-27$ & $\overline{\mathrm{D}}$ & 25.07 .95 & 10 \\
\hline Mark 1147 & $\mathrm{~A}$ & 26.08 .95 & 20 & Mark 816 & B & 13.05 .96 & 20 \\
\hline Mark 971 & B & 01.09 .95 & 20 & PKS $1437-153$ & D & 27.07 .95 & 10 \\
\hline Mark 998 & $\mathrm{~A}$ & 26.08 .95 & 20 & Mark 833 & $\mathrm{C}$ & 22.03 .95 & 20 \\
\hline Q $0155+0220$ & $\mathrm{~B}$ & 31.08 .95 & 20 & Mark 483 & $\mathrm{C}$ & 22.03 .95 & 20 \\
\hline Mark 596 & $\mathrm{~A}$ & 25.08 .95 & 20 & KUV $15519+2144$ & $\mathrm{C}$ & 22.03 .95 & 20 \\
\hline KUV $03079-0101$ & $\mathrm{~A}$ & 27.08 .95 & 20 & Q $1619+3752$ & $\mathrm{~B}$ & 01.09 .95 & 20 \\
\hline \multirow[t]{2}{*}{ CBS 74} & $\mathrm{~A}$ & 30.04 .95 & 15 & EXO $1622.0+2611$ & $\mathrm{C}$ & 22.03 .95 & 20 \\
\hline & $\mathrm{C}$ & 22.03 .95 & 20 & Q $1624+4628$ & $\mathrm{C}$ & 28.08 .95 & 20 \\
\hline HS $0843+2533$ & $\mathrm{C}$ & 10.01 .97 & 20 & Q $1638+4634$ & A & 27.08 .95 & 20 \\
\hline \multirow[t]{2}{*}{ Mark 391} & $\mathrm{~B}$ & 07.01 .97 & 20 & Kaz 110 & B & 23.06 .96 & 20 \\
\hline & $\mathrm{C}$ & 10.01 .97 & 20 & & $\mathrm{C}$ & 22.06 .96 & 20 \\
\hline KUG $0929+324$ & $\mathrm{C}$ & 21.03 .95 & 20 & PKS $1903-80$ & $\mathrm{D}$ & 28.07 .95 & 10 \\
\hline CG 49 & $\mathrm{C}$ & 10.05 .96 & 20 & RN 73 & $\mathrm{~B}$ & 31.08 .95 & 20 \\
\hline UM 446 & $\mathrm{C}$ & 21.03 .95 & 20 & & $\mathrm{C}$ & 30.08 .95 & 20 \\
\hline US 2896 & $\mathrm{C}$ & 22.03 .95 & 20 & Q $2233+0123$ & $\mathrm{C}$ & 30.08 .95 & 20 \\
\hline Mark 646 & $\mathrm{C}$ & 22.03 .95 & 20 & Q $2257+0221$ & $\mathrm{~B}$ & 02.09 .95 & 20 \\
\hline $2 \mathrm{E} 1219+0447$ & $\mathrm{C}$ & 22.03 .95 & 20 & NGC 7678 & $\mathrm{~B}$ & 02.09 .95 & $20^{*}$ \\
\hline KUV $13000+2908$ & $\mathrm{C}$ & 22.03 .95 & 20 & & $\mathrm{C}$ & 30.08 .95 & 20 \\
\hline Q $1356-067$ & $\mathrm{C}$ & 22.03 .95 & 20 & E $2344+184$ & B & 01.09 .95 & 20 \\
\hline \multirow[t]{2}{*}{ Mark 469} & $\mathrm{~B}$ & 08.06 .96 & 20 & UM 11 & $\mathrm{~B}$ & 02.09 .95 & $20^{*}$ \\
\hline & $\mathrm{C}$ & 09.06 .96 & 20 & & $\mathrm{C}$ & 29.08 .95 & 20 \\
\hline
\end{tabular}

1987). In some cases, the classification is based on a single line ratio, either $\lambda 5007 / \mathrm{H} \beta$ or $\lambda 6584 / \mathrm{H} \alpha$. This is potentially dangerous; however we think that in most cases, there is no ambiguity, specially when $\lambda 6584 / \mathrm{H} \alpha<0.3$ (see Fig. 6a in Véron et al. 1997).

\section{Notes on individual objects}

4C 12.05 (Gower et al. 1967) = PKS $0035+121$ (Shimmins et al. 1975) has been tentatively identified by Wills \& Wills (1976) and Jauncey et al. (1978) with a $16.5-17.0$ mag object, the position of which is in good agreement with the accurate radio position measured by Condon et al. (1977); but they have shown that the optical spectrum, although inconclusive, was probably that of a star. However, Wills \& Wills have remarked that there appears to be a small, fainter, south-preceeding blue object, visible on the Palomar Sky Survey prints, blended with the image of the star. A V image, obtained on August 10, 1996 with EFOSC at the $3.6 \mathrm{~m}$ ESO telescope in La Silla shows that, indeed, the object is double, with a separation of 2.8 arcsec. The spectrograph slit was aligned on the two objects $\left(\mathrm{PA}=229^{\circ}\right)$; a 10 min exposure spectrum shows the north-following object to be a star, while the south-preceeding object is a QSO at $z=1.395$ (Fig. 1). The magnitude of the QSO, as measured on the spectrum is about $0.55 \mathrm{mag}$ weaker than the star in $B$, and $0.87 \mathrm{mag}$ in $V$. The emission line fluxes are 570 and 360 $10^{-16} \mathrm{erg} \mathrm{s}^{-1} \mathrm{~cm}^{-2}$ for CIII] $\lambda 1909$ and MgII $\lambda 2798$, respectively.

Mark 1147 is an emission line galaxy (Markarian et al. 1980); it has been erroneously classified as a Seyfert
1 by Véron-Cetty \& Véron (1985). Our low dispersion spectrum (Fig. 2) shows that it is a HII region, with $\lambda 5007 / \mathrm{H} \beta=2.18$ and $\lambda 6584 / \mathrm{H} \alpha=0.24$; this is in agreement with Markarian et al. (1980), who have noticed that $\lambda 6584$ is weak compared to $\mathrm{H} \alpha$.

Mark 971 = KUG 0101+353 (Takase \& MiyauchiIsobe 1991b). Markarian et al. (1984) suggested that it could have an active nucleus; this, however, was not confirmed neither by Denisyuk \& Lipovetski (1984) nor by Lipovetski et al. (1989). Our spectrum (Fig. 6) shows narrow emission lines $\left(<280 \mathrm{~km} \mathrm{~s}^{-1} F W H M\right)$ with $\lambda 5007 / \mathrm{H} \beta=0.41$ together with an $\mathrm{H} \beta$ line in absorption; this object is, therefore, a HII region.

Mark 998. According to Markarian et al. (1984), this galaxy could have an active nucleus; Denisyuk \& Lipovetski (1984) and Lipovetski et al. (1989) were not able to confirm this. Our low dispersion spectrum (Fig. 2) shows $\lambda 6584 / \mathrm{H} \alpha=0.23$. It follows that this object is most probably a HII region.

Q $0155+0220$ is an emission line galaxy according to Schneider et al. (1994). Our spectrum (Fig. 6) shows it to be a HII region with narrow $\left(F W H M<325 \mathrm{~km} \mathrm{~s}^{-1}\right) \mathrm{H} \beta$ and $\lambda 5007$ emission lines, and $\lambda 5007 / \mathrm{H} \beta=0.71$.

Mark 596. This object, having $\lambda 6584 / \mathrm{H} \alpha>1$ may have an active nucleus (Markarian et al. 1984). It is indeed a Seyfert 2 galaxy as our spectrum (Fig. 2) shows that $\lambda 5007 / \mathrm{H} \beta>5$ and $\lambda 6584 / \mathrm{H} \alpha=1.14$.

KUV 03079 - 0101 (Noguchi et al. 1980) is an emission line galaxy according to Chaffee et al. (1991). Our spectrum (Fig. 3) shows broad Balmer lines $\left(3000 \mathrm{~km} \mathrm{~s}^{-1} F H W M\right)$ and narrow [OIII] lines. The ratio of the total $\mathrm{H} \beta$ flux to the $\lambda 5007$ flux is $R=10$; 
Table 3. This table gives for each of the observed objects: the name (Col. 1), the B1950 optical position measured on the Digitized Sky Survey (Cols. 2 and 3), where the the rms error is 0.6 arcsec in each coordinate; "* " indicates objects with larger errors because of their location near one edge of the Schmidt plate (Véron-Cetty \& Véron 1996), the published redshift (Col. 4), the magnitude (Col. 5), the old spectral classification: S2: Seyfert 2, S1: Seyfert 1, HII: HII region, Q: Quasar, ?: unknown (Col. 6), and our classification (Col. 7). References for the finding charts (Col. 8): (1) Anguita et al. (1979), (2) Bolton \& Ekers (1966), (3) Bowen et al. (1994), (4) Condon et al. (1977), (5) Kazarian (1979), (6) Kazarian \& Kazarian (1980), (7) Kondo et al. (1984), (8) MacAlpine \& Williams (1981), (9) MacAlpine et al. (1977), (10) Margon et al. (1985), (11) Markarian \& Lipovetski (1971), (12) Markarian \& Lipovetski (1972), (13) Markarian \& Lipovetski (1973), (14) Markarian \& Lipovetski (1974), (15) Markarian \& Lipovetski (1976), (16) Markarian et al. (1977a), (17) Markarian et al. (1977b), (18) Markarian et al. (1979), (19) Penston (1971), (20) Pesch \& Sanduleak (1983), (21) Pesch \& Sanduleak (1986), (22) Sanduleak \& Pesch (1984), (23) Schneider et al. (1994), (24) Takase \& Miyauchi-Isobe (1985), (25) Wills \& Wills (1976)

\begin{tabular}{|c|c|c|c|c|c|c|c|}
\hline$\overline{\text { Name }}$ & $\alpha$ & $\delta$ & $z$ & mag & old class. & our class. & Ref. \\
\hline $4 \mathrm{C} 12.05$ & 003541.98 & 121103.6 & - & 17.5 & $?$ & $\mathrm{Q}$ & $(25)$ \\
\hline Mark 1147 & 004557.94 & 100356.9 & 0.036 & 15.7 & $\mathrm{~S} 1$ & HII & (18) \\
\hline Mark 971 & 010132.79 & $\begin{array}{lll}35 & 18 & 07.8\end{array}$ & 0.085 & 16.5 & $\mathrm{~S} ?$ & HII & (16) \\
\hline Mark 998 & 013002.04 & -022011.9 & 0.078 & 16.0 & $\mathrm{~S} ?$ & HII & (17) \\
\hline Q $0155+0220$ & 015547.90 & 022026.3 & 0.066 & 16.7 & $?$ & HII & (23) \\
\hline Mark 596 & 024012.67 & $072309.5^{*}$ & 0.038 & 14.8 & $\mathrm{~S} ?$ & $\mathrm{~S} 2$ & (13) \\
\hline KUV $03079-0101$ & 030754.83 & -010110.3 & 0.080 & 16.3 & $?$ & S1.0 & $(7)$ \\
\hline CBS 74 & 082911.46 & 371749.1 & 0.091 & 17. & S & $\mathrm{S} 1.2$ & (21) \\
\hline HS $0843+2533$ & 084356.47 & 253314.7 & 0.050 & 16.8 & $\mathrm{~S} 1$ & $\mathrm{~S} 1$ & - \\
\hline Mark 391 & 085132.38 & 394345.5 & 0.013 & 14.1 & $\mathrm{~S} ?$ & HII & (11) \\
\hline KUG $0929+324$ & 092901.90 & $322659.9^{*}$ & 0.005 & 17.5 & $?$ & HII & (24) \\
\hline CG 49 & 095807.76 & 312644.7 & 0.042 & 16.4 & $\mathrm{~S} 2$ & $\mathrm{~S} 2$ & $(20)$ \\
\hline UM 446 & 113912.06 & -013727.1 & 0.005 & 17.3 & $?$ & HII & $(8)$ \\
\hline US 2896 & 114233.73 & 310356.5 & 0.060 & 16.0 & $\mathrm{~S} 1.5$ & S1 & $(22)$ \\
\hline Mark 646 & 120317.01 & 352727.5 & 0.054 & 15.3 & $\mathrm{~S}$ & S1 & (14) \\
\hline $2 \mathrm{E} 1219+0447$ & 121904.62 & 044704.3 & 0.094 & 16.8 & $?$ & $\mathrm{~S} 1$ & $(3)$ \\
\hline KUV $13000+2908$ & 130001.52 & 290737.0 & 0.023 & 16.1 & $\mathrm{~S} 2$ & HII & (7) \\
\hline Q $1356-067$ & 135644.90 & -060743.8 & 0.072 & 16.2 & $\mathrm{~S} ?$ & HII & - \\
\hline Mark 469 & 141612.98 & 343546.0 & 0.069 & 16.0 & $?$ & HII & $(12)$ \\
\hline PKS $1420-27$ & 141955.50 & -271420.8 & - & 18. & Q? & $\mathrm{Q}$ & $(2)$ \\
\hline Mark 816 & 143140.78 & 525926.8 & 0.089 & 16.5 & $\mathrm{~S} ?$ & HII & $(15)$ \\
\hline PKS $1437-153$ & 143711.31 & -151858.9 & - & 19. & Q? & BL & $(4)$ \\
\hline Mark 833 & 145559.69 & 352405.4 & 0.040 & 16.0 & $\mathrm{~S} ?$ & HII & (15) \\
\hline Mark 483 & 152841.48 & 340553.3 & 0.048 & 16.4 & HII & HII & (12) \\
\hline KUV $15519+2144$ & 155153.33 & 214342.9 & 0.040 & 15.8 & $\mathrm{~S} 2$ & HII & $(7)$ \\
\hline Q $1619+3752$ & 161955.81 & 375236.3 & 0.034 & 17.3 & $?$ & HII & (23) \\
\hline EXO $1622.0+2611$ & 162205.33 & 261123.9 & - & 16.1 & $\mathrm{~S} ?$ & $\mathrm{~S} 1$ & - \\
\hline Q $1624+4628$ & 162434.76 & 462848.2 & 0.030 & 16.1 & $?$ & HII & $(23)$ \\
\hline Q $1638+4634$ & 163850.52 & 463438.8 & 0.059 & 16.4 & $?$ & HII & $(23)$ \\
\hline Kaz 110 & 165716.83 & 690908.0 & 0.053 & 17.2 & HII & HII & $(5)$ \\
\hline PKS $1903-80$ & 190356.16 & -801459.8 & - & 19.0 & $\mathrm{Q} ?$ & $\mathrm{Q}$ & (1) \\
\hline RN 73 & 203608.47 & $\begin{array}{lll}88 & 02 & 05.4\end{array}$ & 0.047 & 17.5 & $?$ & S1.9 & (19) \\
\hline Q $2233+0123$ & 223308.78 & 012400.2 & 0.058 & 16.6 & $?$ & $\mathrm{~S} 1$ & (23) \\
\hline Q $2257+0221$ & 225700.37 & 022129.8 & 0.048 & 16.7 & $?$ & $\mathrm{~S} 2$ & (23) \\
\hline NGC 7678 & 232557.91 & 220844.7 & 0.012 & 15.3 & $\mathrm{~S} 2$ & HII & $(6)^{\prime}$ \\
\hline E $2344+184$ & 234453.30 & 182810.8 & 0.138 & 15.9 & $?$ & $\mathrm{~S} 2$ & (10) \\
\hline UM 11 & 235045.22 & $032622.3^{*}$ & 0.038 & 16.0 & $\mathrm{~S}$ & HII & $(9)$ \\
\hline
\end{tabular}

this object is therefore a Seyfert 1.0 galaxy (Winkler 1992).

CBS 74 is a Seyfert galaxy according to Wagner et al. (1988). It was not detected at $4850 \mathrm{MHz}$ by Gregory \& Condon (1991) $(S<25 \mathrm{mJy})$ and is therefore a radioquiet object. Our spectra (Figs. 3, 9) show that it is a Seyfert 1.2 galaxy with a very broad $\mathrm{H} \alpha$ component $\left(F W H M \sim 12000 \mathrm{~km} \mathrm{~s}^{-1}\right)$ and $R=3.6$. Such broad lines are common in radioloud quasars and broad line radiogalaxies (Miley \& Miller 1979; Wills \& Browne 1986; Brotherton et al. 1994; Eracleous \& Halpern 1994), but they are rare in Seyfert galaxies although a few cases are known, such as 2E 0450-1816 (Eracleous \& Halpern 1994) and Arp 102B (Chen \& Halpern 1989). Indeed, powerful radiogalaxies and radioloud quasars with extended radio morphologies tend to have the broadest Balmer lines, while AGNs with compact radiostructure and radioquiet objects have narrower Balmer lines (Miley \& Miller 1979; Steiner 1981; Wills \& Browne 1986).

HS 0843+2533. The ROSAT X-ray source RX J08469+2522 was identified by Bade et al. (1995) with a 16.8 mag AGN called HS 0843+2533, which exhibits a broad $\mathrm{H} \alpha$ emission line $\left(F W H M=5900 \mathrm{~km} \mathrm{~s}^{-1}\right)$. Our spectrum (Fig. 9) shows, indeed, a strong, broad $\mathrm{H} \alpha$ emission line $\left(F W H M=4850 \mathrm{~km} \mathrm{~s}^{-1}\right)$; this object is therefore a Seyfert 1 galaxy. 


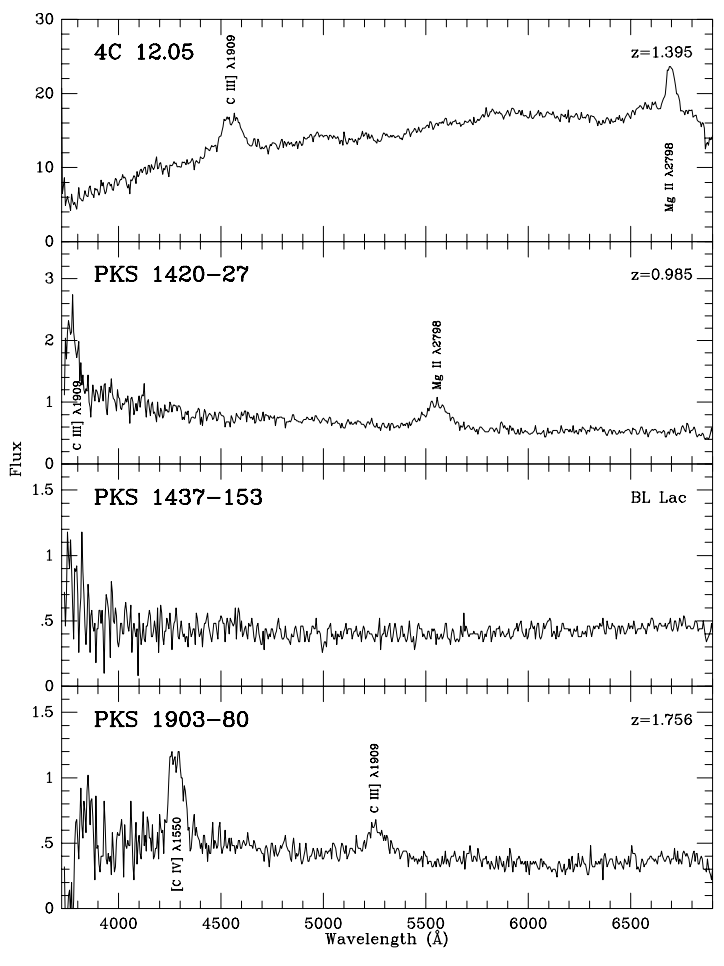

Fig. 1. Low dispersion spectra (resolution $\sim 15 \AA$ ) of four objects observed with the $3.6 \mathrm{~m}$ ESO telescope. The fluxes are in units of $10^{-16} \mathrm{erg} \mathrm{s}^{-1} \mathrm{~cm}^{-2} \AA^{-1}$

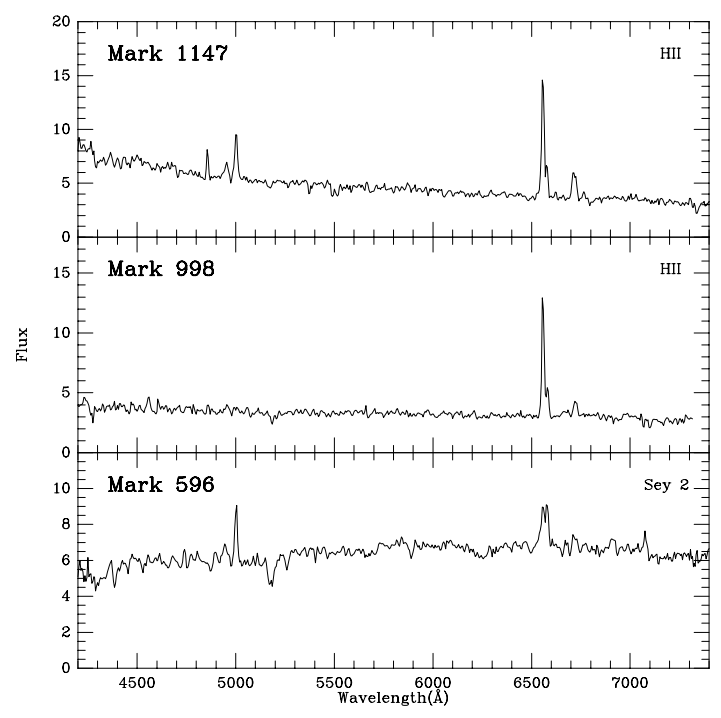

Fig. 2. Low dispersion spectra (resolution $\sim 13.5 \AA$ ), in the rest frame, of three objects observed with the $1.93 \mathrm{~m}$ OHP telescope. The fluxes are in units of $10^{-16} \mathrm{erg} \mathrm{s}^{-1} \mathrm{~cm}^{-2} \AA^{-1}$

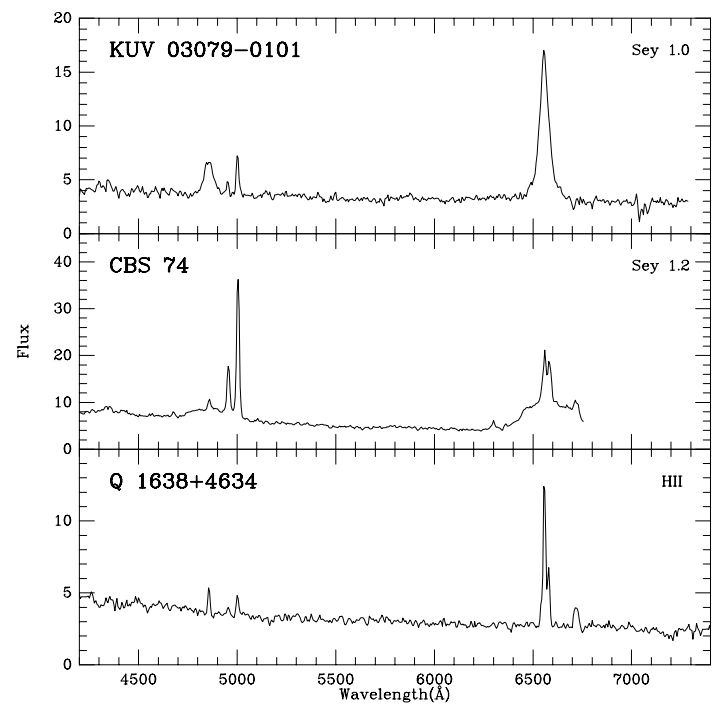

Fig. 3. Same as in Fig. 2 for three additional objects

Mark $391=$ NGC 2691 is a S0a galaxy (Huchra 1977). For Arakelian et al. (1972), it weakly shows the characteristics of the Seyfert nuclei, with a broad $\mathrm{H} \alpha$ emission line ( $~ 50 \AA)$. On this basis, Véron-Cetty \& Véron (1985) classified it as a Seyfert 1. Shuder \& Osterbrock (1981), however, concluded from their own spectroscopic observations that it is not a Seyfert. Our spectra (Fig. 4) show Balmer lines in absorption together with narrow $(F W H M<$ $215 \mathrm{~km} \mathrm{~s}^{-1}$ ) emission lines with $\lambda 5007 / \mathrm{H} \beta=1.21$ and $\lambda 6584 / \mathrm{H} \alpha=0.55$, proving that it is a HII region.

KUG $0929+324$ is a moderate excitation $(\lambda 5007 / \mathrm{H} \beta=2.68)$ emission line galaxy with an heliocentric radial velocity $V=1500 \pm 70 \mathrm{~km} \mathrm{~s}^{-1}$ according to Augarde et al. (1994). Our spectrum (Fig. 7) shows that it is a HII region with narrow emission lines $(F W H M<$ $150 \mathrm{~km} \mathrm{~s}^{-1}$ ) and $\lambda 6584 / \mathrm{H} \alpha=0.10$. We found the radial velocity to be $V=4740 \mathrm{~km} \mathrm{~s}^{-1}$ (Augarde 1995, private communication, gave $\left.V=4478 \mathrm{~km} \mathrm{~s}^{-1}\right)$.

CG 49. Salzer et al. (1995) published line intensity ratios for this object as follows: $\lambda 5007 / \mathrm{H} \beta=11.68$ and $\lambda 6584 / \mathrm{H} \alpha=0.30$. The $\lambda 6584$ line is too strong for a HII region and too weak for a Seyfert 2 galaxy. Our spectrum shows $\lambda 6584$ with the same low intensity, however it so happens that the redshifted wavelength of the $\lambda 6584$ line (6874 $\AA$ ) falls precisely at the position of the atmospheric $B$ band. When corrected for atmospheric absorption by dividing the observed spectrum by the spectrum of a standard star (Fig. 7), we obtain $\lambda 6584 / \mathrm{H} \alpha=0.79$, a normal value for a Seyfert 2 galaxy. The measured $F W H M$ of the emission lines is $\sim 300 \mathrm{~km} \mathrm{~s}^{-1}$, in agreement with the adopted classification.

UM 446 is a moderate excitation $(\lambda 5007 / \mathrm{H} \beta=$ 4.54) emission line galaxy (Salzer et al. 1989). Our spectrum (Fig. 7) shows narrow emission lines $(F W H M<$ 


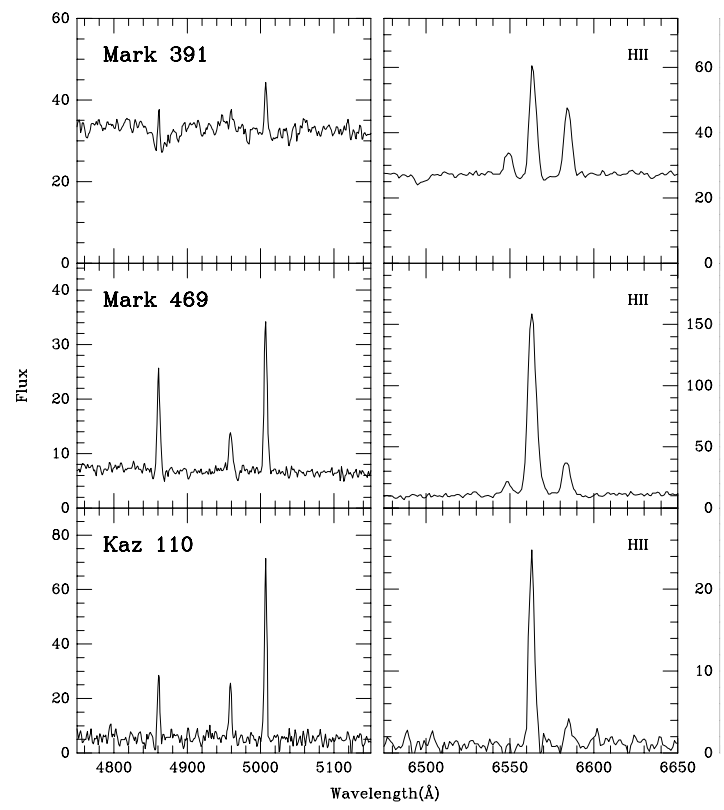

Fig. 4. Blue and red high dispersion spectra (resolution $\sim 3.4 \AA$ ), in the rest frame, of three objects observed with the $1.93 \mathrm{~m}$ OHP telescope. The fluxes are in units of $10^{-16} \operatorname{erg~s}^{-1} \mathrm{~cm}^{-2} \AA^{-1}$

$160 \mathrm{~km} \mathrm{~s}^{-1}$ ) with $\lambda 6584 / \mathrm{H} \alpha=0.04$; this object is therefore a HII region.

US 2896 (Huang \& Usher 1984) = CS 109 (Sanduleak \& Pesch 1984) is an emission line galaxy (Mitchell et al. 1984), and a Seyfert 1.5 galaxy according to Everett \& Wagner (1995). This is confirmed by our spectrum (Fig. 7) which shows a broad $\mathrm{H} \alpha$ component $\left(2100 \mathrm{~km} \mathrm{~s}^{-1} F W H M\right)$. A [OI] $\lambda 6300$ emission line is observed with $\lambda 6300 / \mathrm{H} \alpha_{\text {narrow }}=0.03$.

Mark 646, PG 1203+35 (Green et al. 1986), CG 885 (Pesch \& Sanduleak 1988) or KUG 1203+354 (Takase \& Miyauchi-Isobe 1991a) is a Seyfert galaxy according to Green et al. (1986). Our spectrum (Fig. 7) shows a broad $\mathrm{H} \alpha$ component $\left(2350 \mathrm{~km} \mathrm{~s}^{-1}\right.$ FWHM); Mark 646 is therefore a Seyfert 1 galaxy. A $\lambda 6300$ emission line is observed with $\lambda 6300 / \mathrm{H} \alpha_{\text {narrow }}=0.05$.

2E $\mathbf{1 2 1 9}+\mathbf{0 4 4 7}$ is an emission line galaxy (Bothun et al. 1984; Margon et al. 1985). We classify it as a Seyfert 1 galaxy on the basis of a weak, broad ( FWHM $\sim 8500 \mathrm{~km} \mathrm{~s}^{-1}$ ) $\mathrm{H} \alpha$ component (Fig. 9).

KUV 13000+2908 (Noguchi et al. 1980), CG 963 (Sanduleak \& Pesch 1990) or PB 3241 (Berger et al. 1991) is a Seyfert 2 galaxy according to Wegner \& McMahan (1988). Our spectrum (Fig. 7) shows narrow (FWHM< $185 \mathrm{~km} \mathrm{~s}^{-1}$ ) emission lines with $\lambda 6584 / \mathrm{H} \alpha<0.1$, so this object is a HII region.

Q 1356 - 067 is a QSO according to Goldschmidt et al. (1992). Our spectrum (Fig. 8), however, shows narrow $\left(F W H M<280 \mathrm{~km} \mathrm{~s}^{-1}\right)$ emission lines with

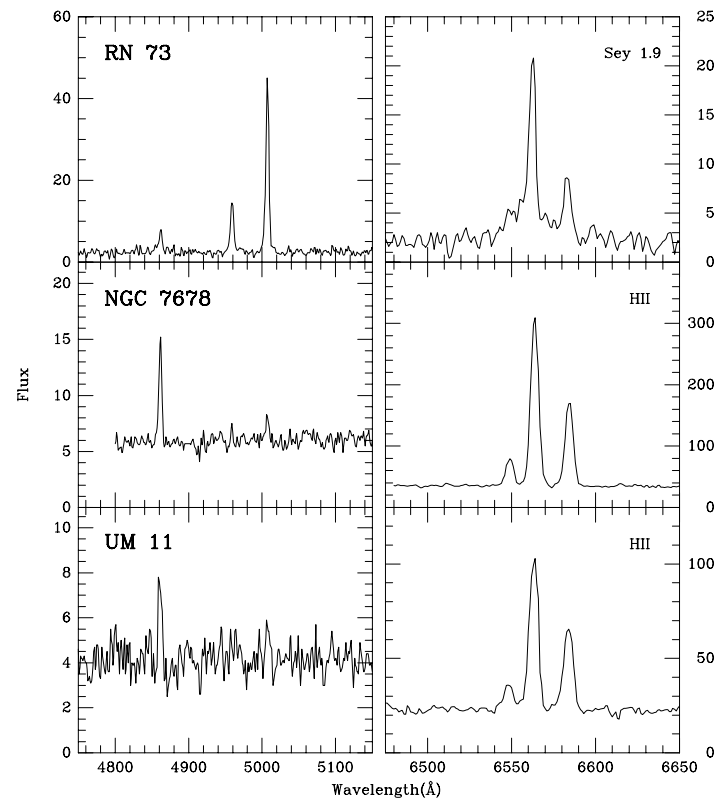

Fig. 5. Same as in Fig. 4 for three additional objects

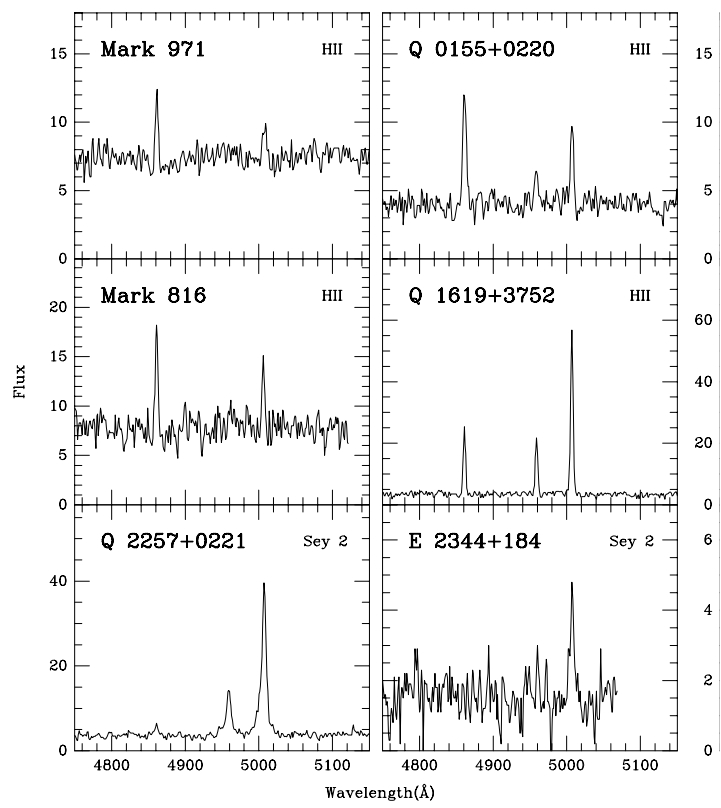

Fig. 6. Blue high dispersion spectra (resolution $\sim 3.4 \AA$ ), in the rest frame, for six objects observed with the $1.93 \mathrm{~m}$ telescope. The fluxes are in units of $10^{-16} \mathrm{erg} \mathrm{s}^{-1} \mathrm{~cm}^{-2} \AA^{-1}$

$\lambda 6584 / \mathrm{H} \alpha=0.16$; this object is therefore a HII region. A $\lambda 6300$ emission line is observed with $\lambda 6300 / \mathrm{H} \alpha=0.015$.

Mark 469, CG 899 (Pesch \& Sanduleak 1989) or KUG $1416+345$ (Takase \& Miyauchi-Isobe 1984) is a 16.1 mag, UV excess galaxy (Peterson et al. 1981). Our observations (Fig. 4) show that it is a HII region, with $\lambda 5007 / \mathrm{H} \beta=1.38$ and $\lambda 6584 / \mathrm{H} \alpha=0.18$, the lines being narrow $\left(F W H M<260 \mathrm{~km} \mathrm{~s}^{-1}\right)$. 


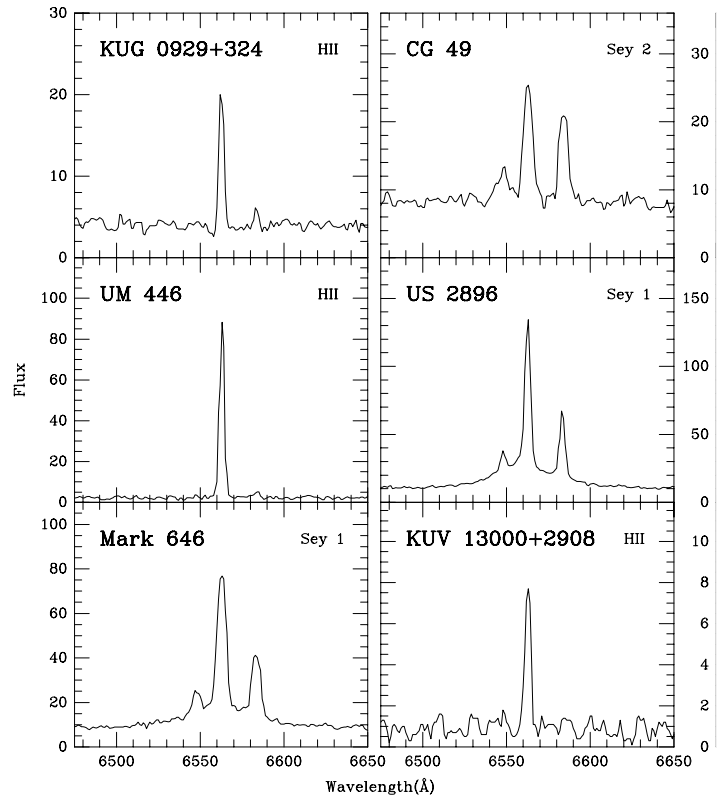

Fig. 7. Red high dispersion spectra (resolution $\sim 3.4 \AA$ ), in the rest frame, for six objects observed with the $1.93 \mathrm{~m}$ telescope. The fluxes are in units of $10^{-16} \mathrm{erg} \mathrm{s}^{-1} \mathrm{~cm}^{-2} \AA^{-1}$

PKS 1420 - 27. This radiosource was identified by Bolton \& Ekers (1966) with an 18 mag QSO. The identification was later confirmed by accurate optical and radio position measurements (Hunstead 1971, 1972). Our spectrum (Fig. 1) shows that it is indeed a QSO at $z=0.985$. The emission line fluxes are 83 and $5310^{-16} \mathrm{erg} \mathrm{s}^{-1} \mathrm{~cm}^{-2}$ for CIII] $\lambda 1909$ and MgII $\lambda 2798$ respectively.

Mark 816 = KUG 1431+529 (Takase \& MiyauchiIsobe 1985) is a $16.5 \mathrm{mag}$, possibly Seyfert, galaxy (Afanasev et al. 1979); however, $\lambda 6584 / \mathrm{H} \alpha<0.3$ (Afanasev et al. 1980). Our spectrum (Fig. 6) shows narrow emission lines $\left(<240 \mathrm{~km} \mathrm{~s}^{-1} F W H M\right)$ with $\lambda 5007 / \mathrm{H} \beta=0.63$. This object is most probably a HII region.

PKS 1437 - 153 is a flat spectrum radiosource identified by Condon et al. (1977) with a 19.0 mag starlike object. It has a featureless spectrum between 3800 and $7000 \AA$ (Fig. 1) and is most probably a BL Lac object.

Mark 833 = CG 590 (Sanduleak \& Pesch 1987) is an emission line galaxy (Markarian et al. 1985) which has been called a "narrow-line active galactic nucleus" by Veilleux \& Osterbrock (1987), based on emission-line intensity ratios published by Shuder \& Osterbrock (1981); it however happened that the object studied in this last paper is Mark 883 which is, in a few occasions mistakenly called Mark 833 (H. Falcke, private communication). The nature of the emission-line nebulosity in Mark 833 was therefore unknown. Our spectrum (Fig. 8) shows narrow $\left(F W H M<225 \mathrm{~km} \mathrm{~s}^{-1}\right)$ emission lines with $\lambda 6584 / \mathrm{H} \alpha=$ 0.30. This object is, therefore, a HII region.

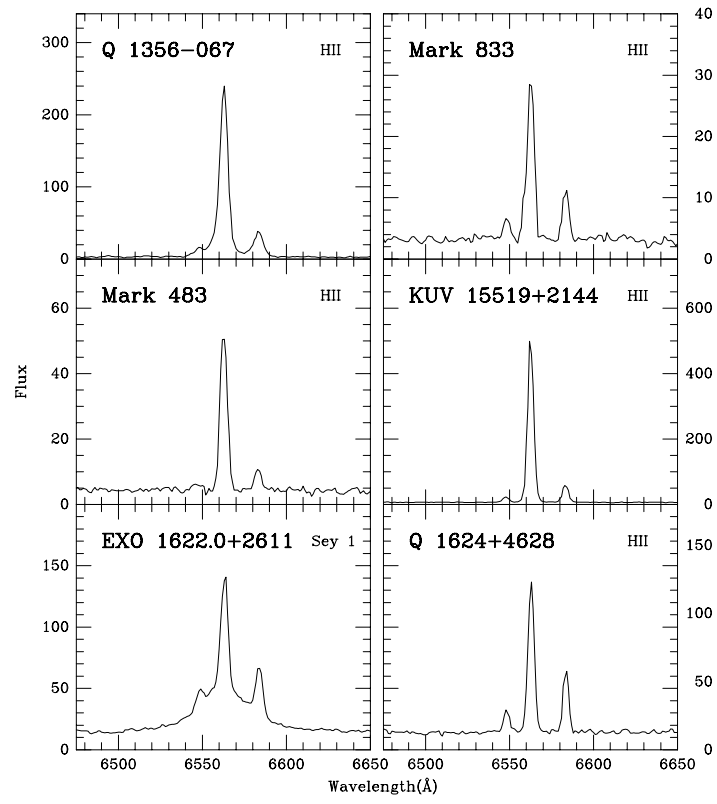

Fig. 8. Same as in Fig. 7 for six additional objects

Mark $483=$ CG 741 (Sanduleak \& Pesch 1987) is an emission line galaxy (Markarian et al. 1988; Izotov et al. 1993) with a strong UV excess $(U-B=-0.45$, Peterson et al. 1981). The emission-line ratios published by Markarian et al. $(\lambda 6584 / \mathrm{H} \alpha<0.33)$ and Izotov et al. $(\lambda 5007 / \mathrm{H} \beta \sim 2.0)$ suggested that it is a HII region. This is confirmed by our spectrum (Fig. 8) which shows narrow emission lines $\left(F W H M<225 \mathrm{~km} \mathrm{~s}^{-1}\right)$ with $\lambda 6584 / \mathrm{H} \alpha=0.12$.

KUV 15519+2144 is a Seyfert 2 galaxy according to Wagner \& Swanson (1990). Our spectrum (Fig. 8) shows it to be a HII region, with narrow $\left(F W H M<185 \mathrm{~km} \mathrm{~s}^{-1}\right)$ emission lines and $\lambda 6584 / \mathrm{H} \alpha=0.11$. A $\lambda 6300$ emission line is observed with $\lambda 6300 / \mathrm{H} \alpha=0.02$

Q 1619+3752, an emission line galaxy according to Schneider et al. (1994), is classified as a HII region, as it shows narrow emission lines $\left(<240 \mathrm{~km} \mathrm{~s}^{-1} F W H M\right)$ and $\lambda 5007 / \mathrm{H} \beta=2.56$ (Fig. 6).

EXO 1622.0+2611. An AGN for Giommi et al. (1991), this is a Seyfert 1 galaxy, as it presents a broad $\mathrm{H} \alpha$ component (1770 $\mathrm{km} \mathrm{s}^{-1}$ FWHM) (Fig. 8).

$\mathrm{Q}$ 1624+4628. An emission line galaxy according to Schneider et al. (1994), it is a HII region, with narrow $\left(<195 \mathrm{~km} \mathrm{~s}^{-1} F W H M\right)$ emission lines and $\lambda 6584 / \mathrm{H} \alpha=$ 0.40 (Fig. 8).

Q 1638+4634. An emission line galaxy according to Schneider et al. (1994), it is a HII region, with $\lambda 5007 / \mathrm{H} \beta=0.86$ and $\lambda 6584 / \mathrm{H} \alpha=0.40$ (Fig. 3).

Kaz 110. The emission-line gas in this object was shown to be ionized by hot stars (Kazarian \& Tamazian 1993). Our spectra (Fig. 4) confirm this result, the measured line ratios being: $\lambda 5007 / \mathrm{H} \beta=2.83$ and $\lambda 6584 / \mathrm{H} \alpha=$ 0.09 . 


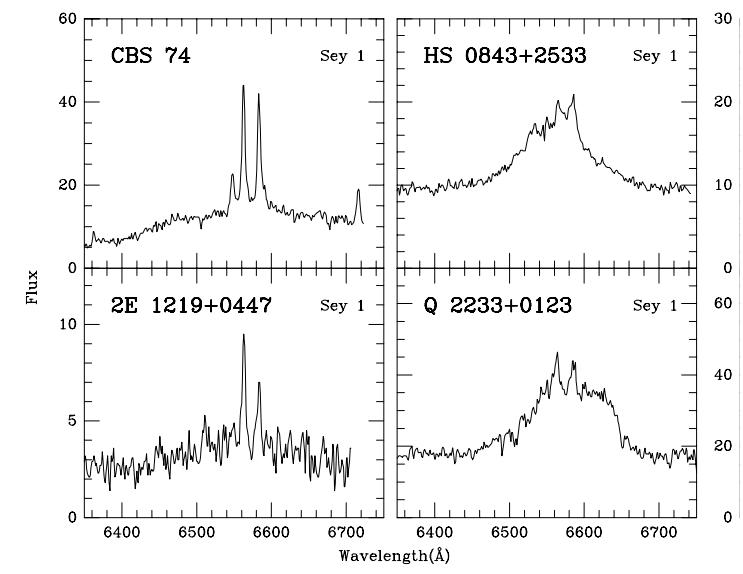

Fig. 9. Same as in Fig. 7 for four additional objects

PKS 1903 - 80. This flat spectrum radiosource (Quiniento \& Cersosimo 1993) was identified with a 19.0 mag QSO by Anguita et al. (1979). The identification was confirmed by an accurate radioposition measurement (Russel et al. 1992). It is indeed a QSO at $z=1.756$ (Fig. 1). The emission line fluxes are 57 and $2510^{-16} \mathrm{erg} \mathrm{s}^{-1} \mathrm{~cm}^{-2}$ for CIV $\lambda 1550$ and CIII] $\lambda 1909$ respectively.

RN 73 (Ryle \& Neville 1962) = 8C 2037+880 (Rees 1990) was identified with a $17.5 \mathrm{mag}$ emission line galaxy (Penston 1971). Our spectra (Fig. 5) show a weak, broad $\left(F W H M \sim 1590 \mathrm{~km} \mathrm{~s}^{-1}\right) \mathrm{H} \alpha$ component, but no broad $\mathrm{H} \beta$ component: this object is a Seyfert 1.9 galaxy. However, the ratio $\lambda 6584 / \mathrm{H} \alpha_{\text {narrow }}=0.37$ is low for a Seyfert galaxy. Halliday (1977) published an accurate radiomap for this source; its position, as measured on this $\operatorname{map}\left(\alpha_{1950}=20^{\mathrm{h}} 36^{\mathrm{m}} 44^{\mathrm{s}}, \delta_{1950}=88^{\circ} 01^{\prime} 58^{\prime \prime}\right)$, is about 20 arcsec away from the position of the galaxy, suggesting that the radiostructure and the galaxy are not to be related.

Q 2233+0123. An emission line galaxy according to Schneider et al. (1994), it is a Seyfert 1, having a strong and broad ( FWHM $\left.\sim 5500 \mathrm{~km} \mathrm{~s}^{-1}\right) \mathrm{H} \alpha$ component (Fig. 9). The profile of this line deviates significantly from a Gaussian, having a flat top.

Q 2257+0221 is an emission line galaxy according to Schneider et al. (1994). It is a Seyfert 2 having broad $\left(F W H M \sim 500 \mathrm{~km} \mathrm{~s}^{-1}\right)$, asymmetrical [OIII] lines, that are much stronger than $\mathrm{H} \beta(\lambda 5007 / \mathrm{H} \beta \sim 17)$ (Fig. 6).

NGC $\mathbf{7 6 7 8}=$ Kaz 336 (Kazarian \& Kazarian 1980). Although classified as a Seyfert 2 galaxy by Kazarian (1993), this is a HII region with $\lambda 6584 / \mathrm{H} \alpha=0.52$, $\lambda 5007 / \mathrm{H} \beta=0.25$ and linewidth $<260 \mathrm{~km} \mathrm{~s}^{-1} F W H M$ (Fig. 5).

E $\mathbf{2 3 4 4}+\mathbf{1 8 4}$ is a spiral galaxy (Hutchings \& Neff 1992). According to Margon et al. (1985), it is an emission line galaxy with a strong $\lambda 6584$ emission line. Our low signal-to-noise blue spectrum (Fig. 6) shows a strong $\lambda 5007$ line $(\lambda 5007 / \mathrm{H} \beta>6)$, so it is most probably a Seyfert 2 galaxy.

UM 11. Terlevich et al. (1991) gave line ratios: $\lambda 6584 / \mathrm{H} \alpha=1.23$ and $\lambda 5007 / \mathrm{H} \beta=0.83$, suggesting that this object is a Liner; our spectra (Fig. 5) give $\lambda 6584 / \mathrm{H} \alpha=0.56$ and $\lambda 5007 / \mathrm{H} \beta \sim 0.5$ (with linewidth $\left.<300 \mathrm{~km} \mathrm{~s}^{-1} F W H M\right)$ showing that it is a HII region instead.

\section{Conclusions}

We have observed 37 AGN candidates and classified them on the basis of their spectroscopic properties; the line intensities and widths were obtained by fitting the spectra with Gaussian components. We concluded that three of the observed objects are confirmed QSOs, one is a BL Lac object, nine are Seyfert 1 galaxies, four are Seyfert 2s, while twenty are HII regions.

Acknowledgements. We are grateful to Dr. M. Viton for having allowed us to take the low dispersion spectrum of CBS 74 during his observing run. This research has made use of the NASA/IPAC extragalactic database (NED) which is operated by the Jet Propulsion Laboratory, Caltech, under contract with the National Aeronautics and Space Administration. A.C. Gonçalves acknowledges support from the JNICT during the course of this work, in the form of a PRAXIS XXI PhD. grant (PRAXIS XXI/BD/5117/95).

\section{References}

Afanasev V.L., Denisyuk E.K., Lipovetski V.A., 1979, Soviet Astron. Lett. 5, 144

Afanasev V.L., Lipovetski V.A., Markarian B.E., Stepanian D.A., 1980, Astrophys. 16, 119

Anguita C., Campusano L.E., Torres C., Pedreros M., 1979, AJ 84, 718

Arakelian M.A., Dibai E.A., Esipov V.F., 1972, Astrophys. 8, 197

Augarde R., Chalabaev A., Comte G., Kunth D., Machara H., 1994, A\&AS 104, 259

Bade N., Fink H.H., Engels D., et al., 1995, A\&AS 110, 649

Berger J., Cordoni J.P., Fringant A.-M., et al., 1991, A\&AS 87,389

Bolton J.G., Ekers J., 1966, Aust. J. Phys. 19, 275

Bothun F.D., Margon B., Balick B., 1984, PASP 96, 583

Bowen D.V., Osmer S.J., Blades J.C., et al., 1994, AJ 107, 481

Brotherton M.S., Wills B.J., Steidel C.C., Sargent W.L.W., 1994, ApJ 423, 131

Chaffee F.H., Foltz C.B., Hewett P.C., et al., 1991, AJ 102, 461

Chen K., Halpern J.P., 1989, ApJ 344, 115

Condon J.J., Hicks P.D., Jauncey D.L., 1977, AJ 82, 692

Dekker H., D’Odorico S., Arsenault R., 1988, A\&A 189, 353

Denisyuk E.K., Lipovetski V.A., 1984, Astrophys. 20, 290

Eracleous M., Halpern J.P., 1994, ApJS 90, 1

Everett M.E., Wagner R.M., 1995, PASP 107, 1059

Giommi P., Tagliaferri G., Beuermann K., et al., 1991, ApJ 378,77

Goldschmidt P., Miller L., La Franca F., Cristiani S., 1992, MNRAS 256, 65P 
Table 4. Fitting profile analysis results (see text). Column 2 gives the redshift used to deredshift the spectra, while Cols. 3 and 7 give the velocity of the lines as measured on the dereshifted spectra. Columns 6 and 10 give the peak intensity of the $\mathrm{H} \beta$ and $\mathrm{H} \alpha$ lines respectively (in units of $10^{-16} \mathrm{erg} \mathrm{s}^{-1} \mathrm{~cm}^{-2} \AA^{-1}$ ). The $F W H M$ are observed values, not corrected for the instrumental profile; values in parenthesis are from low dispersion spectra and are unresolved

\begin{tabular}{|c|c|c|c|c|c|c|c|c|c|c|}
\hline$\overline{\text { Name }}$ & $\bar{z}$ & $\begin{array}{c}V \\
\left(\mathrm{~km} \mathrm{~s}^{-1}\right)\end{array}$ & $\begin{array}{c}F W H M \\
\left(\mathrm{~km} \mathrm{~s}^{-1}\right)\end{array}$ & $\frac{\lambda 5007}{\mathrm{H} \beta}$ & $\overline{\mathrm{H} \beta}$ & $\begin{array}{c}V \\
\left(\mathrm{~km} \mathrm{~s}^{-1}\right)\end{array}$ & $\begin{array}{c}F H W M \\
\left(\mathrm{~km} \mathrm{~s}^{-1}\right)\end{array}$ & $\frac{\lambda 6584}{\mathrm{H} \alpha}$ & $\mathrm{H} \alpha$ & $\begin{array}{l}\text { Spectral } \\
\text { type }\end{array}$ \\
\hline Mark 1147 & 0.0364 & 14 & $(780)$ & 2.18 & 2.3 & 11 & $(635)$ & 0.24 & 11.4 & HII \\
\hline Mark 971 & 0.0823 & 15 & 280 & 0.41 & 5.6 & - & - & - & - & HII \\
\hline Mark 998 & 0.0761 & - & - & - & - & 22 & $(580)$ & 0.23 & 10.3 & HII \\
\hline Q $0155+0220$ & 0.0651 & -1 & 325 & 0.71 & 8.4 & - & - & - & - & HII \\
\hline Mark 596 & 0.0388 & -16 & $(960)$ & $>5.0$ & $<0.6$ & 45 & $(720)$ & 1.14 & 2.2 & $\mathrm{~S} 2$ \\
\hline \multirow[t]{2}{*}{ KUV $03079-0101$} & 0.0807 & -15 & $(860)$ & $>10.0$ & $<0.4$ & - & - & - & - & S1.0 \\
\hline & & -29 & 3360 & - & 3.1 & -28 & 2570 & - & 12.2 & \\
\hline \multirow[t]{2}{*}{ CBS 74} & 0.0920 & 143 & $(945)$ & 15.6 & 1.9 & -10 & 260 & 0.89 & 27.8 & $\mathrm{~S} 1.2$ \\
\hline & & 1485 & 14500 & - & 2.3 & 1092 & 12200 & - & 10.4 & \\
\hline \multirow[t]{2}{*}{ HS $0843+2533$} & 0.0507 & - & - & - & - & 90 & 475 & 1.6 & 2.5 & $\mathrm{~S} 1$ \\
\hline & & - & - & - & - & -70 & 4850 & - & 7.3 & \\
\hline Mark 391 & 0.0133 & 9 & 210 & 1.21 & 9.9 & 45 & 225 & 0.55 & 36.7 & HII \\
\hline KUG $0929+324$ & 0.0158 & - & - & - & - & -6 & 150 & 0.10 & 17.6 & HII \\
\hline CG 49 & 0.0438 & - & - & - & - & 15 & 300 & 0.79 & 17.4 & $\mathrm{~S} 2$ \\
\hline UM 446 & 0.0061 & - & - & - & - & 9 & 160 & 0.04 & 79.8 & HII \\
\hline \multirow[t]{2}{*}{ US 2896} & 0.0594 & - & - & - & - & -3 & 185 & 0.43 & 103 & $\mathrm{~S} 1$ \\
\hline & & - & - & - & - & -120 & 2100 & - & 12.3 & \\
\hline \multirow[t]{2}{*}{ Mark 646} & 0.0536 & - & - & - & - & 0 & 280 & 0.46 & 62.8 & $\mathrm{~S} 1$ \\
\hline & & - & - & - & - & -16 & 2350 & - & 8.0 & \\
\hline \multirow[t]{2}{*}{$2 \mathrm{E} 1219+0447$} & 0.0947 & - & - & - & - & 3 & 225 & 0.54 & 5.5 & $\mathrm{~S} 1$ \\
\hline & & - & - & - & - & 222 & 8440 & - & 1.6 & \\
\hline KUV $13000+2908$ & 0.0223 & - & - & - & - & 3 & 185 & $<0.1$ & 7.0 & HII \\
\hline Q $1356-067$ & 0.0746 & - & - & - & - & 6 & 280 & 0.16 & 226 & HII \\
\hline Mark 469 & 0.0689 & -9 & 295 & 1.38 & 19.4 & 18 & 260 & 0.18 & 147 & HII \\
\hline Mark 816 & 0.0887 & -19 & 240 & 0.63 & 10.5 & - & - & - & - & HII \\
\hline Mark 833 & 0.0395 & - & - & - & - & -8 & 225 & 0.30 & 25.7 & HII \\
\hline Mark 483 & 0.0481 & - & - & - & - & -5 & 225 & 0.12 & 48.3 & HII \\
\hline KUV $15519+2144$ & 0.0392 & - & - & - & - & -9 & 185 & 0.11 & 483 & HII \\
\hline Q $1619+3752$ & 0.0331 & -3 & 240 & 2.56 & 20.4 & - & - & - & - & HII \\
\hline \multirow[t]{2}{*}{ EXO $1622.0+2611$} & 0.0394 & - & - & - & - & 15 & 225 & 0.40 & 93.0 & $\mathrm{~S} 1$ \\
\hline & & - & - & - & - & 12 & 1770 & - & 33.0 & \\
\hline Q $1624+4628$ & 0.0301 & - & - & - & - & 6 & 195 & 0.40 & 124 & HII \\
\hline Q $1638+4634$ & 0.0581 & 10 & $(720)$ & 0.86 & 1.8 & 36 & $(625)$ & 0.40 & 10.3 & HII \\
\hline Kaz 110 & 0.0527 & -5 & 225 & 2.83 & 22.9 & 9 & 170 & 0.09 & 23.1 & HII \\
\hline \multirow[t]{2}{*}{ RN 73} & 0.0491 & 18 & 310 & 7.4 & 5.4 & -16 & 185 & 0.37 & 16.3 & S1.9 \\
\hline & & - & - & - & - & -34 & 1590 & - & 3.1 & \\
\hline \multirow[t]{2}{*}{ Q $2233+0123$} & 0.0566 & - & - & - & - & 15 & 290 & 0.60 & 8.5 & $\mathrm{~S} 1$ \\
\hline & & - & - & - & - & 1080 & 5500 & - & 21.3 & \\
\hline Q $2257+0221$ & 0.0466 & 3 & 495 & 16.7 & 1.9 & - & - & - & - & $\mathrm{S} 2$ \\
\hline NGC 7678 & 0.0116 & 12 & 270 & 0.25 & 9.5 & 45 & 250 & 0.52 & 272 & HII \\
\hline E $2344+184$ & 0.1365 & 24 & 425 & $>6.0$ & $<0.5$ & - & - & - & - & $\mathrm{S} 2$ \\
\hline UM 11 & 0.0390 & -8 & 310 & 0.46 & 3.8 & 21 & 290 & 0.56 & 80.2 & HII \\
\hline
\end{tabular}

Gower J.F.R., Scott P.F., Wills D., 1967, Mem. RAS 71, 49 Green R.F., Schmidt M., Liebert J., 1986, ApJS 61, 305

Gregory P.C., Condon J.J., 1991, ApJS 75, 1011

Halliday J., 1977, MNRAS 179, 111

Huang K.L., Usher P.D., 1984, ApJS 56, 393

Huchra J.P., 1977, ApJS 35, 171

Hunstead R.W., 1971, MNRAS 152, 277

Hunstead R.W., 1972, MNRAS 157, 367

Hutchings J.B., Neff S.G., 1992, AJ 104, 1

Izotov Y.I., Lipovestski V.A., Guseva N.G., et al., 1993, A\&A Trans. 3, 197

Jauncey D.L., Wright A.E., Peterson B.A., Condon J.J., 1978, ApJ 219, L1

Kazarian M.A., 1979, Astrophys. 15, 1

Kazarian M.A., 1993, Astrophys. 36, 217

Kazarian M.A., Kazarian E.S., 1980, Astrophys. 16, 7
Kazarian M.A., Tamazian V.S., 1993, Astrophys. 36, 222

Kondo M., Noguchi T., Machara H., 1984, Ann. Tokyo Astron. Obs. 20, 130

Lemaître G., Kohler D., Lacroix D., Meunier J.-P., Vin A., 1989, A\&A 228, 546

Lipovetski V.A., Shapavalova A.I., Stepanian D.A., Erastova L.K., 1989, Astrophys. 31, 665

MacAlpine G.M., Williams G.A., 1981, ApJS 45, 113

MacAlpine G.M., Smith S.B., Lewis D.W., 1977, ApJS 34, 95

Margon B., Downes R.A., Chanan G.A., 1985, ApJS 59, 23

Markarian B.E., Lipovetski V.A., 1971, Astrophys. 7, 299

Markarian B.E., Lipovetski V.A., 1972, Astrophys. 8, 89

Markarian B.E., Lipovetski V.A., 1973, Astrophys. 9, 283

Markarian B.E., Lipovetski V.A., 1974, Astrophys. 10, 185

Markarian B.E., Lipovetski V.A., 1976, Astrophys. 12, 429 
Markarian B.E., Lipovetski V.A., Stepanian D.A., 1977a, Astrophys. 13, 116

Markarian B.E., Lipovetski V.A., Stepanian D.A., 1977b, Astrophys. 13, 215

Markarian B.E., Lipovetski V.A., Stepanian D.A., 1979, Astrophys. 15, 130

Markarian B.E., Lipovetski V.A., Stepanian D.A., 1980, Astrophys. 16, 1

Markarian B.E., Lipovetski V.A., Stepanian D.A., 1984, Astrophys. 20, 581

Markarian B.E., Erastova L.K., Lipovetski V.A., Stepanian D.A., Shapovalova A.I., 1985, Astrophys. 22, 127

Markarian B.E., Erastova L.K., Lipovetski V.A. Stepanian D.A., Shapovalova A.I., 1988, Astrophys. 28, 14

Massey P., Strobel K., Barnes J.V., Anderson E., 1988, ApJ 328,315

Miley G.K, Miller J.S., 1979, ApJ 228, L55

Mitchell K.J., Warnock A., Usher P.D., 1984, ApJ 287, L3

Noguchi T., Machara H., Kondo M., 1980, Ann. Tokyo Astron. Obs. 18,55

Oke J.B., 1974, ApJS 27, 21

Oke J.B., Gunn J.E., 1983, ApJ 266, 713

Penston M.V., 1971, ApJ 170, 395

Pesch P., Sanduleak N., 1983, ApJS 51, 171

Pesch P., Sanduleak N., 1986, ApJS 60, 543

Pesch P., Sanduleak N., 1988, ApJS 66, 297

Pesch P., Sanduleak N., 1989, ApJS 70, 163

Peterson B.M., Fricke K., Biermann P., 1981, PASP 93, 281

Quiniento Z.M., Cersosimo J.C., 1993, A\&AS 97, 435

Rees N., 1990, MNRAS 244, 233

Russel J.L., Jauncey D.L., Harvey B.R., et al., 1992, AJ 103, 2090

Ryle M., Neville A.C., 1962, MNRAS 125, 39

Salzer J.J., MacAlpine G.M., Boroson T.A., 1989, ApJS 70, 447
Salzer J.J., Moody J.W., Rosenberg J.L., Gregory S.A., Newberry M.V., 1995, AJ 109, 2376

Sanduleak N., Pesch P., 1984, ApJS 55, 517

Sanduleak N., Pesch P., 1987, ApJS 63, 809

Sanduleak N., Pesch P., 1990, ApJS 72, 291

Schneider D.P., Schmidt M.S., Gunn J.E., 1994, AJ 107, 1245

Shimmins A.J., Bolton J.G., Wall J.V., 1975, Aust. J. Phys. Astrophys. Suppl. 34, 63

Shuder J.M., Osterbrock D.E., 1981, ApJ 250, 55

Steiner J.E., 1981, ApJ 250, 469

Stone R.P.S., 1977, ApJ 218, 767

Takase B., Miyauchi-Isobe N., 1984, Ann. Tokyo Astron. Obs. 19,595

Takase B., Miyauchi-Isobe N., 1985, Ann. Tokyo Astron. Obs. 21,127

Takase B., Miyauchi-Isobe N., 1991a, Pub. Nat. Obs. Japan 2, 37

Takase B., Miyauchi-Isobe N., 1991b, Pub. Nat. Obs. Japan 2, 239

Terlevich R., Melnick J., Masegosa J., Moles M., Copetti M.V.F., 1991, A\&AS 91, 285

Veilleux S., Osterbrock D.E., 1987, ApJS 63, 295

Véron-Cetty M.-P., Véron P., 1985, ESO Scientific Report No. 4

Véron-Cetty M.-P., Véron P., 1986, A\&AS 65, 241

Véron-Cetty M.-P., Véron P., 1996, A\&AS 115, 97

Véron P., Gonçalves A.C., Véron-Cetty M.-P., 1997, A\&A 319, 52

Wagner R.M., Swanson S.R., 1990, AJ 99, 330

Wagner R.M., Sion E.M., Liebert J., Stanfield S.G., 1988, ApJ 328,213

Wegner G., McMahan R.K., 1988, AJ 96, 1933

Wills B.J., Browne I.W.A., 1986, ApJ 302, 56

Wills D., Wills B.J., 1976, ApJS 31, 143

Winkler H., 1992, MNRAS 257, 677 